\title{
Using Item Response Theory for Explainable Machine Learning in Predicting Mortality in the Intensive Care Unit: Case-Based Approach
}

Adrienne Kline ${ }^{1,2,3}, \mathrm{PhD}$; Theresa Kline ${ }^{4}, \mathrm{MSc}, \mathrm{PhD}$; Zahra Shakeri Hossein Abad ${ }^{3,5}, \mathrm{MSc}, \mathrm{PhD}$; Joon Lee ${ }^{3,5,6}, \mathrm{PhD}$

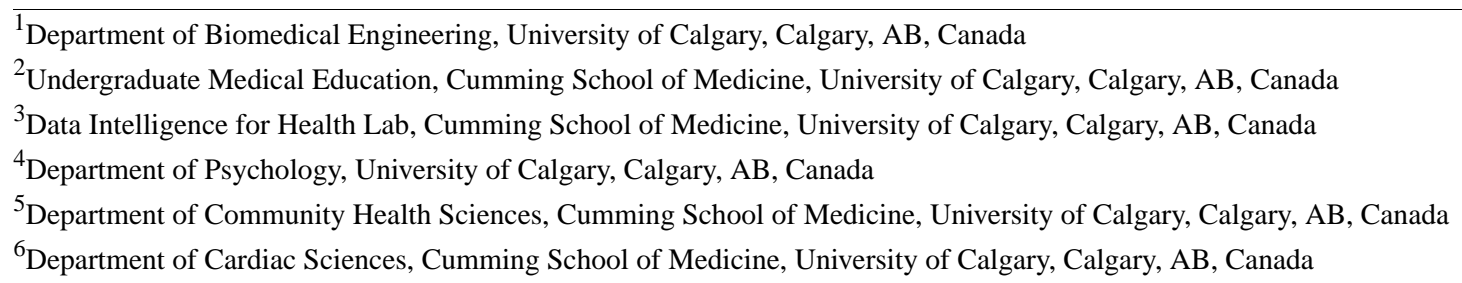

Corresponding Author:

Adrienne Kline, $\mathrm{PhD}$

Department of Biomedical Engineering

University of Calgary

2500 University Drive NW

Calgary, $\mathrm{AB}$

Canada

Phone: 15875831725

Email: askline1@gmail.com

\section{Abstract}

Background: Supervised machine learning (ML) is being featured in the health care literature with study results frequently reported using metrics such as accuracy, sensitivity, specificity, recall, or F1 score. Although each metric provides a different perspective on the performance, they remain to be overall measures for the whole sample, discounting the uniqueness of each case or patient. Intuitively, we know that all cases are not equal, but the present evaluative approaches do not take case difficulty into account.

Objective: A more case-based, comprehensive approach is warranted to assess supervised ML outcomes and forms the rationale for this study. This study aims to demonstrate how the item response theory (IRT) can be used to stratify the data based on how difficult each case is to classify, independent of the outcome measure of interest (eg, accuracy). This stratification allows the evaluation of ML classifiers to take the form of a distribution rather than a single scalar value.

Methods: Two large, public intensive care unit data sets, Medical Information Mart for Intensive Care III and electronic intensive care unit, were used to showcase this method in predicting mortality. For each data set, a balanced sample ( $\mathrm{n}=8078$ and $\mathrm{n}=21,940$, respectively) and an imbalanced sample ( $\mathrm{n}=12,117$ and $\mathrm{n}=32,910$, respectively) were drawn. A 2-parameter logistic model was used to provide scores for each case. Several ML algorithms were used in the demonstration to classify cases based on their health-related features: logistic regression, linear discriminant analysis, K-nearest neighbors, decision tree, naive Bayes, and a neural network. Generalized linear mixed model analyses were used to assess the effects of case difficulty strata, ML algorithm, and the interaction between them in predicting accuracy.

Results: The results showed significant effects $(P<.001)$ for case difficulty strata, ML algorithm, and their interaction in predicting accuracy and illustrated that all classifiers performed better with easier-to-classify cases and that overall the neural network performed best. Significant interactions suggest that cases that fall in the most arduous strata should be handled by logistic regression, linear discriminant analysis, decision tree, or neural network but not by naive Bayes or K-nearest neighbors. Conventional metrics for ML classification have been reported for methodological comparison.

Conclusions: This demonstration shows that using the IRT is a viable method for understanding the data that are provided to ML algorithms, independent of outcome measures, and highlights how well classifiers differentiate cases of varying difficulty. This method explains which features are indicative of healthy states and why. It enables end users to tailor the classifier that is appropriate to the difficulty level of the patient for personalized medicine. 
(J Med Internet Res 2020;22(9):e20268) doi: 10.2196/20268

\section{KEYWORDS}

item response theory; machine learning; statistical model; mortality

\section{Introduction}

\section{Background}

This study aims to demonstrate an approach to assess the effectiveness of binary machine learning (ML) classification, which is an alternative to the more traditional single scalar measures in the literature. Our approach uses an item response theory (IRT) model to enhance the understanding of the data set on which ML protocols are run as well as the results of the classification outcomes. Aspects of IRT's utility have recently surfaced in the ML literature, including comparisons of collaborative filtering [1], evaluation of natural language processing systems [2], identification of initial computer adaptive learning items [3], assessment of the utility of ML classifiers [4], and ML feature selection [5]. However, using IRT in the manner proposed in this study has not yet been undertaken.

The varied and numerous contexts (eg, business, finances, medicine, home, government agencies) in which ML is being used is no less than staggering [6]. Since the advent of the development of ML classification protocols, there has been a commensurate interest in assessing and comparing their efficacy [7]. Evaluation techniques fall into several major categories [8-12]. Many techniques use estimates of single scalar values to summarize the quality of classification based on the frequencies in a confusion matrix (true-positive, false-positive, true-negative, and false-negative). The most common measures include accuracy, precision, negative predictive value, sensitivity, and specificity, although some combine sensitivity and specificity (eg, Youden index, likelihoods, and discriminant power) [13]. Further refinements of scalar estimates have been introduced including reducing the amount of bias and variance of the estimate that have enhanced their interpretability [14], presenting statistical comparisons (eg, $t$ test, conservative $\mathrm{z}$, McNemar test) between ML protocol scalar outcomes [15] and assessing the invariance of estimates with changes in the confusion matrix [16]. Graphical presentations of the confusion matrix data at various points along a continuum include gain and lift charts, receiver operating characteristic curves, and area under the curve (AUC). These provide a more comprehensive depiction of the various scalar measures [12] by contextualizing them.

Despite the advances in metric development, there is an interest in developing more extensive descriptions of ML classification outcomes. For example, it has been argued that "... any single scalar measure has significant limitations" and "that such measures ... oversimplify complex questions and combine things that should be kept separate" [17]. Another issue is that many different programs, search and optimize strategies, and evaluation approaches have populated the literature, sparking researchers to attempt to systematize the findings for more general consumption [18]. Some reviews have supported the contention that ML algorithms should outperform human experts
[19], but others have found that overly complex approaches used in some ML models are no better than simpler, more intuitive models [20]. Studies should give readers an understanding of the reasons why algorithms perform differently, rather than simply providing results of differences between 2 scalar summary values [21,22].

These comments are consistent with general calls for a fuller explanation regarding the interpretability of ML studies [23], particularly in the biomedical and health fields that have been slower to exploit this technology. Overreliance on text-based information rather than contextual elements and the inherent uncertainty in medical decision making have been cited as problems in applying ML findings [24]. The associations within data sets that are theory-free offer little guidance with regard to improving clinical care [25]. Medical professionals are not well trained in how ML works and thus are not able to critically evaluate the utility of the reported findings [26].

The ML criticism of the lack of attention to the unique characteristics of the individual cases is the focus of this study. We propose to address this challenge using a more comprehensive, case-nuanced approach. Although there has been some work in this regard, such as the now accepted wisdom that standard classifiers do not work well with imbalanced data [27], a focus on the individual cases that fall into the miss or false-positive categories has only rarely been investigated as a point of interest [28].

This lack of attention is highlighted in the assessment study of various ML models; they often result in comparable outcomes as similar percentages of cases are misclassified regardless of the model used [29]. Some insight into this phenomenon was brought to light in a study on benchmarking data sets, where some sets had more difficult-to-classify cases and other sets contained largely easy-to-classify cases, providing similar results at the aggregate level regardless of the ML approach used [30]. It has been argued that cleaning up the data by eliminating some cases in the training data set is an appropriate tactic to improve classification accuracy [31]. We disagree with this approach and instead argue that hard-to-classify cases should be examined in a more systematic manner. This study shifts the focus from the level of utility of an ML only at the aggregate sample level toward pinpointing where that model falls short and, more importantly, why the model falls short. The process described identifies, a priori, which cases in the data set (balanced or imbalanced) will be more or less difficult to classify. The evaluation of ML algorithms across these cases will help to understand why these cases are difficult to classify. Examining this phenomenon in detail is as important as the classification accuracy index of the data set as a whole.

There are 2 fundamental building blocks to any ML system: the features of interest and the cases in the data set. To investigate the research question in this study, methods derived from IRT were employed as they simultaneously estimate the 
characteristics of both features and cases. Understanding this phenomenon allows medical professionals to tailor the classifier to the patient.

\section{Similarities Between ML and Test Taking}

Following an examination, there are discussions by students about the test items; often they remark "that question was hard, what did you put?" or "that was as easy question." Such comments reflect the purposeful construction of the test items. Some items are designed to be relatively easy to pass whereas others are designed to be more difficult such that only a few can pass. Similarly, students talk about the test takers- "she always gets the highest score in the class" or "I think I have a 50-50 chance of passing." Test takers are quite cognizant of the fact that not all test items are created equal and that not all test takers have the same ability. These fundamental assumptions give rise to IRT, where the characteristics of the items and of the students are modeled together, providing a clearer picture about which items discriminate between which test takers.

A parallel can be drawn between a set of students passing or failing a test based on their performance on a set of items with a set of patients being classified into 1 of 2 categories (alive or not alive) based on their scores on a set of health-related features. Using the test item information, an ML classifier should be able to predict which students will pass or fail the test, and using the feature information, an ML classifier should be able to predict which patients will be alive or not alive. There is likely to be a base level at which it correctly classifies cases as belonging to 1 group or another by chance alone, and additional case information on each feature should enhance the prediction. Some cases can be easily partitioned into the pass the test (ie, they pass all the items) or fail the test (ie, they fail all the items). Cases with more moderate levels of mastery however would be expected to pass some and fail some items (features in ML terms). It is these difficult cases where classifiers would be less likely to successfully predict their probability of passing or failing the test. One option to enhance prediction is to add more features to help classify these more difficult cases, but doing so results in high dimensionality, overfitting models, difficult-to-interpret findings, and nongeneralizing results. This quandary is a classic optimization problem in the ML literature.

As not all test takers score $100 \%$ or $0 \%$ on an examination, some combination of right and wrong answers to questions provides an index of individual test-taker ability in completing the test. The term ability (symbolized by the term theta, $\theta$ ) is used in the psychometric literature where IRT evolved and is used to describe any latent construct of interest being measured. In this study, within-range or out-of-range laboratory values and vital signs as well as demographic information comprise the features in our data sets. Thus, we can ascertain a case's placement with respect to the underlying distribution of unhealthiness. These individual case-based indices create a distribution of unhealthiness across all features (or in our case laboratory values, vital signs, and demographic information). Depending on where individual patients fall on the distribution, the ease with which ML classifiers correctly predict the outcome (mortality) is expected to be affected-those concentrated in the central area of the distribution will be more challenging to correctly classify relative to those cases lying more at the tails of the distribution. Thus, rather than labeling case scores as being on a healthy-unhealthy continuum, suggesting these scores might only be useful in a health context, we use the classification difficulty index (CDI) because of their ease or difficulty in being able to be correctly classified using supervised ML.

The process of generating CDIs on the unhealthiness continuum will be carried out without using the outcome variable of mortality itself, that is, IRT provides case-based scores (CDIs) that can be examined before the data as a collective is subjected to an ML protocol.

\section{Specific Study Hypotheses and Research Questions}

The IRT analysis provides case-based CDIs using a set of feature characteristics that do not use the information on the outcome classification variable. CDIs for the sample are generated along the normal distribution, with a mean 0.0 (SD 1.0). It is hypothesized that cases with more centrally located CDIs will be less likely to be classified correctly, whereas cases with more peripherally located CDIs will be more likely to be classified correctly. One research question is as follows: Will some ML classifiers be more accurate in classifying cases at all CDIs? Another research question is as follows: Will some ML classifiers be more accurate than others in classifying cases at different CDIs? Identifying these cases a priori provides an alternative manner to evaluate different ML protocols or classification methods and will advance our understanding of ML findings and the data they are being fed with.

\section{Methods}

\section{Data Sets}

Data were obtained through 2 large, freely available data sets. One was the MIMIC-III (Medical Information Mart for Intensive Care III) database housing health data of $>40,000$ critical care unit patients at the Beth Israel Deaconess Medical Center admitted between 2001 and 2012 [32,33]. The other was the electronic intensive care unit (eICU) Collaborative Research Database that houses data from critical care unit patients from across the continental United States admitted between 2014 and 2015 [34].

\section{Case Inclusion}

Databases were queried using the SQL plug-in for Python (Python Software Foundation). Case inclusion criteria were as follows: (1) age 16 years, (2) at least three-fourth of the features of interest were available for a select case (patient), leading to subsequent imputation, and (3) first hospital visit in the case of repeated patients. Features of predictive interest were selected based on 2 common severity of illness scores: Simplified Acute Physiology Score II and Acute Physiology and Chronic Health Evaluation IV for MIMIC-III and eICU, respectively. To test the hypothesis with both balanced and imbalanced data sets, the number of death cases in both data sets (coded 1) was noted and the same number of cases of no death was then randomly selected and incorporated into the balanced data sets. Imbalanced data sets were created by randomly sampling twice as many no death cases compared with death cases. We used the 1/3:2/3 
imbalance ratio to detect any change in results using a somewhat mildly imbalanced than an extremely imbalanced set.

For the MIMIC-III data set, there were 4039 cases that experienced death in hospital, resulting in a final and balanced sample size of 8078 and imbalanced sample size of 12,117. In the eICU data set, there were 10,970 death in hospital cases. Employing the same methodology resulted in a balanced sample size of 21,940 and an imbalanced sample size of 32,910.

\section{Features}

The features included demographic, procedural, pre-existing conditions, and laboratory values (Tables 1 and 2). Normal values were presented and were obtained from the Medical Council of Canada [35] unless otherwise noted. Laboratory values represent the worst values taken during the intensive care unit (ICU) stay in both data sets in the first 24 hours. In the IRT component of the analyses, variables were dichotomized into disease-promoting states (1) akin to failing the item on the test or disease-protective states ( 0 ; passing the item). Values that fell outside the normal laboratory ranges were coded as 1 (too low or too high). Pre-existing conditions were coded as 1 (present) or 0 (absent). Age was demarcated at 65 years, with those aged $>65$ years coded 1 and those aged $\leq 65$ years coded 0 [36-39]. For the sex variable, men were assigned 1 and women were assigned 0 [40]. Imputation of missing data was performed using a multiple imputation chained equations technique using the impyute library in Python 3.7.7 to preserve the pre-existing distribution of features.

Table 1. Medical Information Mart for Intensive Care III variables based on Simplified Acute Physiology Score II.

\begin{tabular}{|c|c|c|}
\hline Feature name & Description & Normal values, units \\
\hline AIDS & Pre-existing diagnosis & Absent: 0,0 or 1 \\
\hline Heme malignancy & Pre-existing diagnosis & Absent: 0,0 or 1 \\
\hline Metastatic cancer & Pre-existing diagnosis & Absent: 0,0 or 1 \\
\hline Minimum GCS ${ }^{\mathrm{a}}$ & Glasgow Coma Scale & $15^{\mathrm{b}}, 1-15$ \\
\hline $\mathrm{WBC}^{\mathrm{c}}$ minimum & Lowest white blood cell & $4-10,10^{9}$ \\
\hline WBC maximum & Highest white blood cell & $4-10,10^{9}$ \\
\hline Na minimum & Sodium minimum & $135-145, \mathrm{mmol} / \mathrm{L}$ \\
\hline Na maximum & Sodium maximum & $135-145, \mathrm{mmol} / \mathrm{L}$ \\
\hline $\mathrm{K}$ minimum & Potassium minimum & $3.5-5, \mathrm{mmol} / \mathrm{L}$ \\
\hline $\mathrm{K}$ maximum & Potassium maximum & $3.5-5, \mathrm{mmol} / \mathrm{L}$ \\
\hline Bilirubin maximum & Bilirubin maximum & $\leq 1.52, \mathrm{mg} / \mathrm{dL}$ \\
\hline $\mathrm{HCO}_{3}$ minimum & Bicarbonate minimum & $24-30, \mathrm{mmol} / \mathrm{L}$ \\
\hline $\mathrm{HCO}_{3}$ maximum & Bicarbonate maximum & $24-30, \mathrm{mmol} / \mathrm{L}$ \\
\hline$B U N^{d}$ minimum & Blood urea nitrogen minimum & $7-22, \mathrm{mg} / \mathrm{dL}$ \\
\hline BUN maximum & Blood urea nitrogen maximum & $7-22, \mathrm{mg} / \mathrm{dL}$ \\
\hline $\mathrm{PO}_{2}$ & Partial pressure of oxygen & 85-105, mm Hg \\
\hline $\mathrm{FiO}_{2}$ & Fraction of inspired oxygen & $21, \%$ \\
\hline Heart rate mean & Mean heart rate & $60-100, \mathrm{bpm}$ \\
\hline BP mean & Mean systolic blood pressure & 95-145, mm Hg \\
\hline Max temp & Maximum temperature & $36.5-37.5,{ }^{\circ} \mathrm{C}$ \\
\hline Urine output & Urine output & $800-2000^{\mathrm{e}}, \mathrm{mL} / 24 \mathrm{~h}$ \\
\hline Sex & Male or female & Male: 1 , Female: 0 , Male or female \\
\hline Age & Age in years & $\leq 65: 0$, years \\
\hline Admission type & Emergency or elective & Emergency: 1 ; else: $0, \mathrm{~N} / \mathrm{A}^{\mathrm{f}}$ \\
\hline
\end{tabular}

${ }^{\mathrm{a}}$ GCA: Glasgow Coma Scale.

${ }^{\mathrm{b}}$ Teasdale and Jennett, 1974 [41]; Teasdale and Jennett, 1976 [42].

${ }^{\mathrm{c}} \mathrm{WBC}$ : white blood cell.

${ }^{\mathrm{d}} \mathrm{BUN}$ : blood urea nitrogen.

${ }^{\mathrm{e}}$ Medical CMP, 2011 [43].

${ }^{\mathrm{f}} \mathrm{N} / \mathrm{A}$ : not applicable. 
Table 2. Electronic intensive care unit data set variables based on Acute Physiology and Chronic Health Evaluation IV.

\begin{tabular}{|c|c|c|}
\hline Feature name & Description & Normal values, Units \\
\hline $\mathrm{GCS}^{\mathrm{a}}$ & Glasgow Coma Scale & $15^{\mathrm{b}}, 1-15$ \\
\hline Urine output & Urine output in 24 hours & $800-2000^{\mathrm{c}}, \mathrm{mL} / 24$ hour \\
\hline $\mathrm{WBC}^{\mathrm{d}}$ & White blood cell count & $4-10,10^{9}$ \\
\hline $\mathrm{Na}$ & Serum sodium & $135-145, \mathrm{mmol} / \mathrm{L}$ \\
\hline Temperature & Temperature in Celsius & $36.5-37.5^{\mathrm{e}},{ }^{\circ} \mathrm{C}$ \\
\hline Respiration rate & Highest white blood cell & $12-20^{\mathrm{f}}$, breaths $/ \mathrm{min}$ \\
\hline Heart rate & Heart rate/min & $60-100^{\mathrm{f}}, \mathrm{bpm}$ \\
\hline Mean blood pressure & Mean arterial pressure & $70-100^{\mathrm{g}}, \mathrm{mm} \mathrm{Hg}$ \\
\hline Creatinine & Serum creatinine & $0.57-1.02\left(\mathrm{~F}^{\mathrm{h}}\right) ; 0.79-1.36\left(\mathrm{M}^{\mathrm{i}}\right), \mathrm{mEq} / \mathrm{L}$ \\
\hline $\mathrm{pH}$ & Arterial pH & $7.35-7.45, \mathrm{~N} / \mathrm{A}^{\mathrm{j}}$ \\
\hline Hematocrit & Red blood cell volume & $37-46(\mathrm{~F}) ; 38-50(\mathrm{M}), \%$ \\
\hline Albumin & Serum albumin & $3.5-5.0, \mathrm{~g} / \mathrm{dL}$ \\
\hline $\mathrm{PO}_{2}$ & Partial pressure of oxygen & $85-105, \mathrm{~mm} \mathrm{Hg}$ \\
\hline $\mathrm{PCO}_{2}$ & Partial pressure carbon dioxide & $35-45, \mathrm{~mm} \mathrm{Hg}$ \\
\hline $\mathrm{BUN}^{\mathrm{k}}$ & Blood urea nitrogen maximum & $7-22, \mathrm{mg} / \mathrm{dL}$ \\
\hline Glucose & Blood sugar level & $68-200, \mathrm{~mL} / \mathrm{dL}$ \\
\hline Bili & Serum bilirubin & $\leq 1.52, \mathrm{md} / \mathrm{dL}$ \\
\hline $\mathrm{FiO}_{2}$ & Fraction of inspired oxygen & $21^{1}, \%$ \\
\hline Sex & Male or female & Male: 1 ; female: $0, \mathrm{M}$ or $\mathrm{F}$ \\
\hline Age & Age in years & $\leq 65: 0$, years \\
\hline Leukemia & Pre-existing diagnosis & Absent: 0,0 or 1 \\
\hline Lymphoma & Pre-existing diagnosis & Absent: 0,0 or 1 \\
\hline Cirrhosis & Pre-existing diagnosis & Absent: 0,0 or 1 \\
\hline Hepatic failure & Pre-existing diagnosis & Absent: 0,0 or 1 \\
\hline Metastatic cancer & Pre-existing diagnosis & Absent: 0,0 or 1 \\
\hline AIDS & Pre-existing diagnosis & Absent: 0,0 or 1 \\
\hline Thrombolytics & Medical intervention & Absent: 0,0 or 1 \\
\hline Ventilator & Medical intervention & Absent: 0,0 or 1 \\
\hline Dialysis & Medical intervention & Absent: 0,0 or 1 \\
\hline Immunosuppressed & Medical intervention & Absent: 0,0 or 1 \\
\hline Elective surgery & Medical intervention & Absent: 0,0 or 1 \\
\hline
\end{tabular}

${ }^{\mathrm{a} G C S}$ : Glasgow Coma Scale.

${ }^{\mathrm{b}}$ Teasdale and Jennett, 1974 [41]; Teasdale and Jennett, 1976 [42].

${ }^{\mathrm{c}}$ Medical CMP, 2011 [43].

${ }^{\mathrm{d}}$ WBC: white blood cell.

e Lapum et al. 2018 [44].

${ }^{\mathrm{f}}$ MDCalc [45].

${ }^{\mathrm{g}}$ Healthline [46]

${ }^{\mathrm{h}} \mathrm{F}$ : female.

${ }^{\mathrm{i}} \mathrm{M}$ : male.

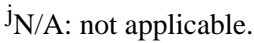


${ }^{\mathrm{k}}$ BUN: blood urea nitrogen.

${ }^{1}$ eICU Collaborative Research Database [47].

\section{IRT Analyses}

Using the IRTPRO (Scientific Software International) program, a 2-parameter logistic model (2PL) was run on the dichotomous data. The program uses a marginal maximum likelihood estimation procedure to calculate feature and case parameters [48] and assumes that respondents belong to a population that can be characterized by their placement on a latent normal probability distribution-unhealthiness in this study with the left and right sides of the distribution indicating better and worse health, respectively [49]. Although higher scores on the latent distribution in IRT usually indicate better outcomes (eg, students have passed more items on a test), in this context, higher scores mean more of the patients' features were out of range and are thus associated with worse outcomes (ie, higher likelihood of death). The output generates logistic item characteristic curves that describe each feature's relationship to the underlying distribution. For each feature, 2 characteristics were estimated, slope and location.

Equation 1 shows a 2PL model in IRT; slope $\left(\mathrm{a}_{\mathrm{i}}\right)$ captures the discriminability capacity of the feature. Feature functions with flat slopes indicate that they are not very discriminatory, whereas those with steep slopes are highly discriminatory, particularly at the inflection point. The location $\left(b_{i}\right)$ denotes where along the function the inflection point occurs. As the functions are set along the standard normal distribution (mean 0.0, SD 1.0), this point indicates where along the unhealthiness continuum the feature is most likely to differentiate cases. An example is presented in Figure 1.

Figure 1. Characteristic curve using a 2-parameter logistic model.

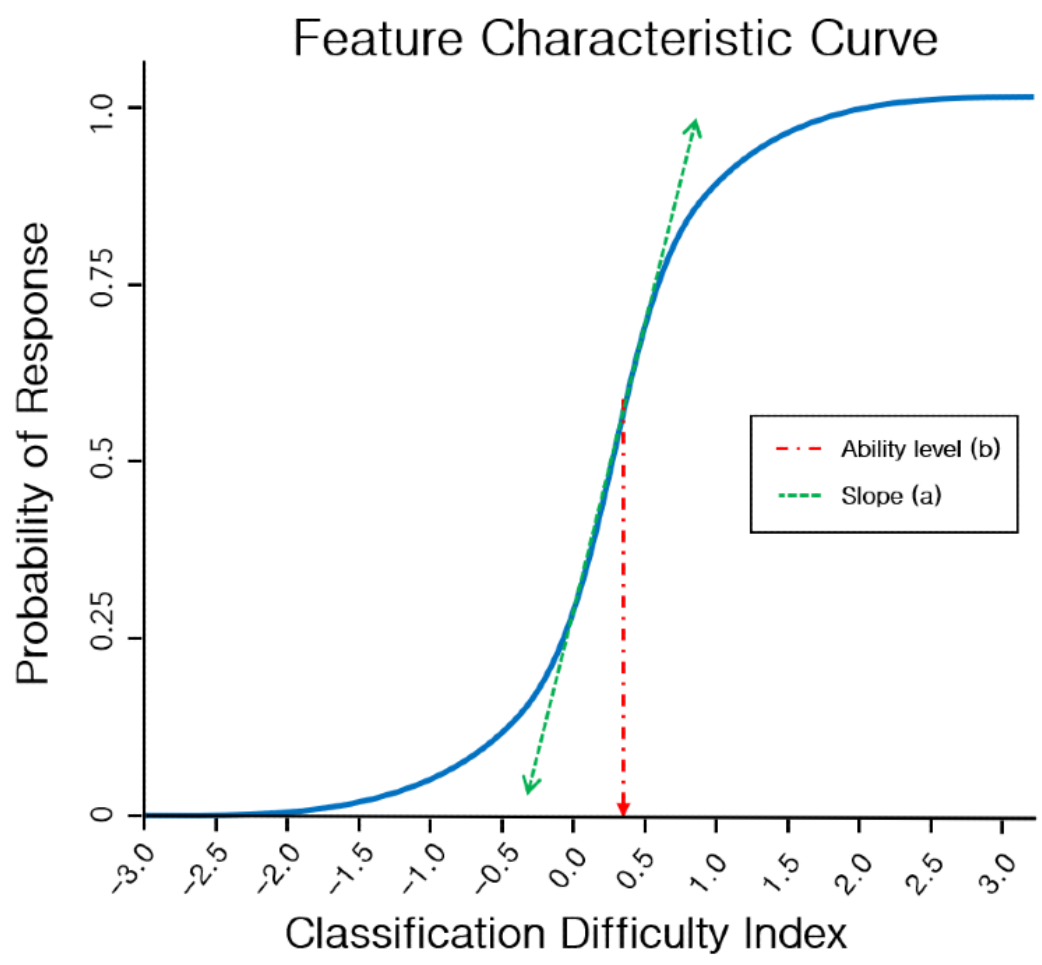

$$
P_{i j}\left(\theta_{j}, b_{i}, a_{i}\right)=\frac{\exp \left[a_{i}\left(\theta_{j}-b_{i}\right)\right]}{1+\exp \left[a_{i}\left(\theta_{j}-b_{j}\right)\right]}
$$

CDI estimation in a 2PL model is calculated based on equation 2 , where the probability of obtaining the correct answer is based on the scores on the items' $u_{i}$ weighted by $a_{i}$.

$$
\begin{gathered}
\sum_{i} a_{i} P\left(\hat{\theta}, b_{i}, a_{i}\right)=\sum_{i} a_{i} u_{i}{ }^{(2)} \\
L(\theta)=\prod_{i} P_{i}\left(\theta, a_{i}, b_{i}\right)^{u_{i}} Q_{i}\left(\theta, a_{i}, b_{i}\right)^{1-u_{i} Z}
\end{gathered}
$$

Equation 3, where $u_{i} \in(0,1)$ is the score on item $i$, is called the likelihood function. It is the probability of a response pattern given the CDIs and the item parameters across cases. There is 1 likelihood function for each response pattern, and the sum of all such functions equals 1 at any value of the distribution. On the basis of the pattern of each case's values on the features, the program uses a Bayesian estimation process that provides a CDI on the unhealthiness continuum for each case in the data set.

CDIs are reported on the standard normal distribution and typically range between -2.50 and +2.50 . Each case's CDI has its own individual SE around it based on the individual's pattern of results across all features and their unique characteristics. Using the results from the 2 PL model, it was possible to identify which of the cases were more centrally or more peripherally 
located on the distribution and thus would be less or more likely to be accurately classified into their respective categories (no death or death).

To allow for easy visualization and testing of effects, several strata bins were created into which continuous IRT CDIs could be assigned. These bins were separated at every 0.5 difficulty change in the distribution of the data. The first bin was centered over the 0.0 mark to denote the most difficult cases and subsequent bins were demarcated at 0.5 levels toward each periphery. This process of bin allocation continued until all observed CDIs for the cases were accounted for.

\section{Analyses}

Multiple ML algorithms were tested using the original feature values for both MIMIC-III and eICU data sets. These included logistic regression, linear discriminant analysis, K-nearest neighbors, decision tree, naive Bayes, and neural network. Both the K-nearest neighbors and neural network had their hyperparameters optimized by a grid search. In the case of the $\mathrm{K}$-nearest neighbors, the search grid included $\mathrm{K}$ from 1 to 40 and distance methods of Minkowski, Hamming, and Manhattan. The grid investigated for the neural network included activation functions such as softmax, softplus, softsign, relu, Tanh, sigmoid, and hard sigmoid; learning rates such as $0.001,0.01$, $0.1,0.2$, and 0.3 ; and hidden neurons in a single hidden layer of $1,5,10,15,20,25$, and 30 . In each of these methods, a 10 -fold cross-validation was performed, and the numerical prediction was extracted for each case and then reassociated with its subject ID number for graphical plotting. The evaluation methods, accuracy, precision, recall, F1, and AUC metrics were calculated. Accuracy was used to assess the hypotheses and research questions.

\section{Comparison Analyses}

To test the main effects of CDI and the repeated measure of the ML classifier as well as their interaction on each case's accuracy score $(0,1)$, generalized linear mixed model (GLMM) [50] analyses were conducted using the GENLINMIX program of
SPSS 23 [51]. GENLINMIX uses the penalized quasi-likelihood estimation method for fixed effects. Separate analyses for each of the balanced and imbalanced data sets were conducted. The standard form of the GLMM is shown in equations 4 and 5. y is a response vector, and $\mathrm{b}$ is the random effects vector. Distr is a conditional distribution of $y$ given $b . \mu$ is the conditional mean, and is the dispersion parameter.

In equation $5, g(\mu)$ is the logit link function that defines the relationship between the mean response $\mu$ and the linear combination of predictors. $X$ represents the fixed effects matrix, and $\mathrm{Z}$ is a random effects matrix, where is simply an offset to the model.

$$
\begin{aligned}
& y_{i} \mid b \sim \operatorname{Distr}\left(\mu_{i}, \frac{\sigma^{2}}{w_{i}}\right) \\
& g(\mu)=X \beta+Z b+\delta{ }^{(5)}
\end{aligned}
$$

The models specified that (1) all effects are fixed, (2) the dependent variable follows a binomial distribution, and thus the predictors and criterion are linked via a logit function, (3) the residual covariance matrix for the repeated measure (ML classifier) is diagonal, and (4) the reference category was set to 0 . Follow-up paired-comparison tests on the estimated marginal and cell means used a $P$ level of $<.001$ to protect against a type I error.

\section{Results}

\section{IRT 2PL Model Results}

Descriptive results of case CDIs are shown in Table 3, and frequency distributions are shown in Figures 2 and 3 (MIMIC-III) and Figures 4 and 5 (eICU).

It should be noted that the 2 data sets have different distributions, and this fingerprint is inherently unique to the data set processed.

\begin{tabular}{|c|c|c|c|c|c|c|c|c|}
\hline \multirow[t]{2}{*}{ Data set } & \multirow[t]{2}{*}{$\mathrm{CDI}^{\mathrm{a}}$ range } & \multirow[t]{2}{*}{ Overall, mean (SD) } & \multicolumn{2}{|c|}{ Point-biserial correlations ${ }^{\mathrm{b}}$} & \multirow{2}{*}{$\begin{array}{l}\text { No death, } \\
\text { mean (SD) }\end{array}$} & \multirow{2}{*}{$\begin{array}{l}\text { Death, mean } \\
\text { (SD) }\end{array}$} & \multicolumn{2}{|c|}{ Two-tailed $t$ value $^{\mathrm{c}}$} \\
\hline & & & $r$ value & $P$ value & & & $t$ test $(\mathrm{df})$ & $P$ value \\
\hline $\begin{array}{l}\text { MIMIC-III }{ }^{\mathrm{d}} \text { bal- } \\
\text { anced }\end{array}$ & $\begin{array}{l}-1.81 \text { to } \\
+2.16\end{array}$ & $0.00(0.85)$ & 0.37 & $<.001$ & $-0.32(0.79)$ & $0.32(0.80)$ & $35.76(8077)$ & $<.001$ \\
\hline $\begin{array}{l}\text { MIMIC-III imbal- } \\
\text { anced }\end{array}$ & $\begin{array}{l}-1.70 \text { to } \\
+2.27\end{array}$ & $0.00(0.85)$ & 0.35 & $<.001$ & $-0.21(0.80)$ & $0.42(0.80)$ & $\begin{array}{l}40.88 \\
(12116)\end{array}$ & $<.001$ \\
\hline $\mathrm{eICU}^{\mathrm{e}}$ balanced & $\begin{array}{l}-2.63 \text { to } \\
+2.83\end{array}$ & $0.00(0.80)$ & 0.50 & $<.001$ & $-0.40(0.73)$ & $0.40(0.64)$ & $\begin{array}{l}86.18 \\
(21939)\end{array}$ & $<.001$ \\
\hline eICU imbalanced & $\begin{array}{l}-2.55 \text { to } \\
+2.93\end{array}$ & $0.00(0.81)$ & 0.51 & $<.001$ & $-0.29(0.73)$ & $0.59(0.61)$ & $\begin{array}{l}109.09 \\
(32909)\end{array}$ & $<.001$ \\
\hline
\end{tabular}

Table 3. Item response theory case classification difficulty index results.

${ }^{\mathrm{a}} \mathrm{CDI}$ : classification difficulty index.

${ }^{\mathrm{b}}$ Between CDI and outcome (no death or death).

${ }^{\mathrm{c}}$ Difference between no death and death means.

${ }^{d}$ MIMIC III: Medical Information Mart for Intensive Care.

eICU: electronic intensive care unit. 
Figure 2. Classification Difficulty Indexes in MIMIC-III (A) balanced and (B) imbalanced data. CDI: classification difficulty index; MIMIC: Medical Information Mart for Intensive Care.

A

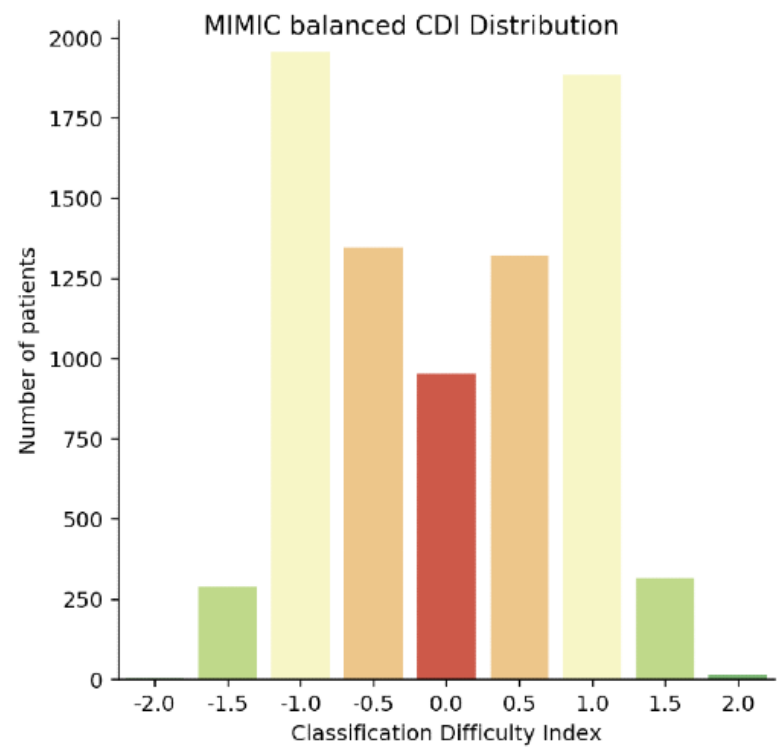

B

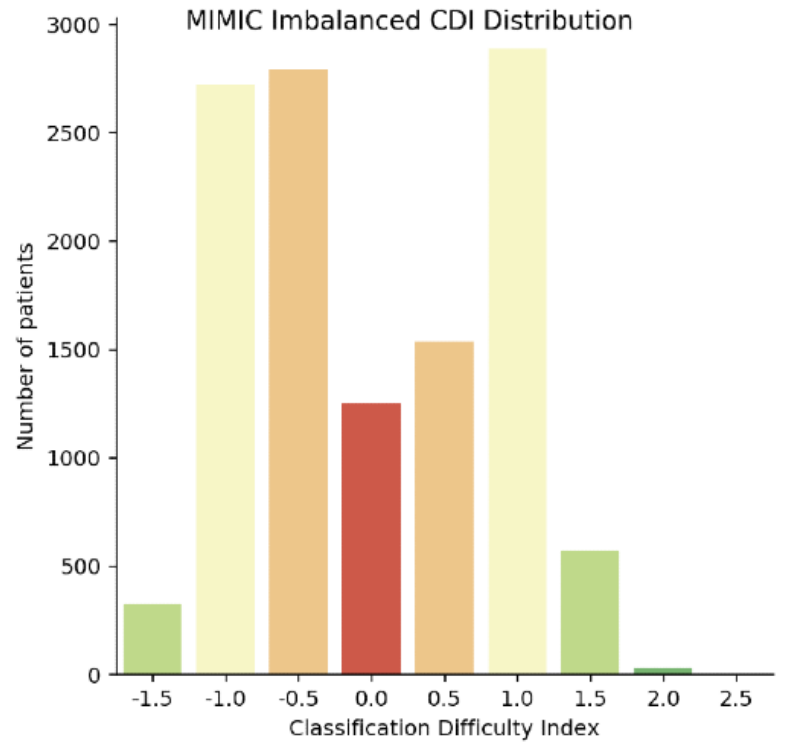

Figure 3. Classification Difficulty Indexes in eICU (A) balanced and (B) imbalanced data. eICU: electronic Intensive Care Unit; DT: decision tree; KNN: K-nearest neighbors; LDA: linear discriminant analysis; LR: logistic regression; NB: naive Bayes; NN: neural network.

A

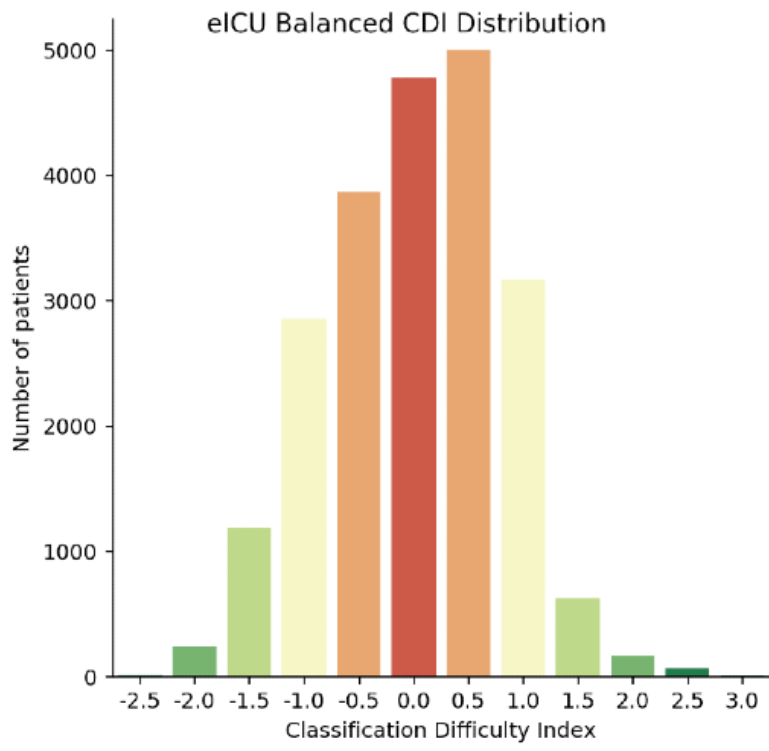

Using the feature parameter estimates and case CDI, the unique differentiating capacity for each feature can be depicted by calculating the probability of each case falling into the 0 (no death) or 1 (death) categories. For example, the slope and location parameters for the blood urea nitrogen (BUN) minimum
B

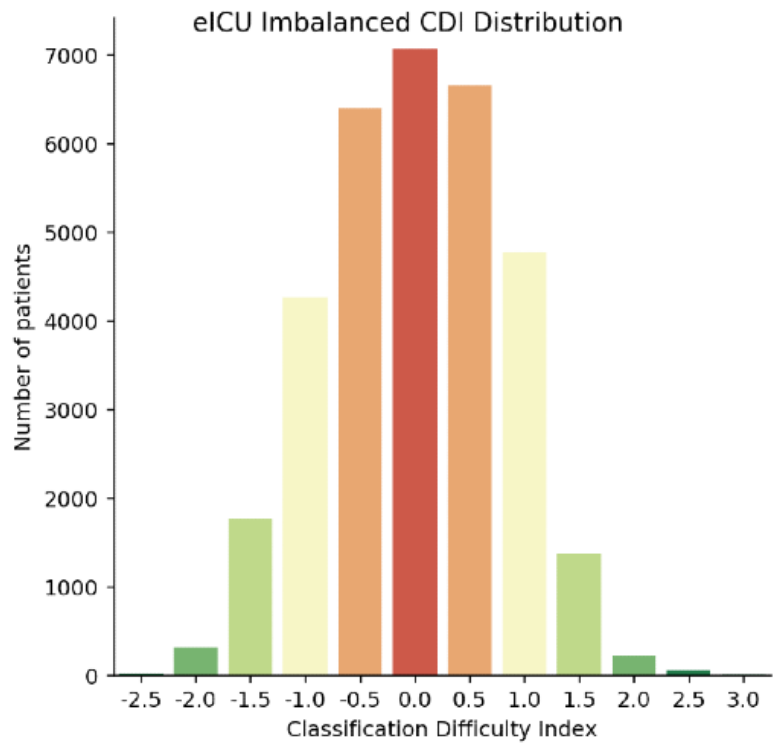

and urine output for the 2 MIMIC-III data sets are shown in Table 4. The higher slope of the BUN minimum feature is contrasted with the very low slope of the urine output feature. These differences highlight the importance of some features over others in terms of being useful in categorizing cases. 
Table 4. Medical Information Mart for Intensive Care III feature parameters.

\begin{tabular}{llc}
\hline Feature parameters & Slope & Location \\
\hline Balanced & 5.64 & 0.09 \\
$\quad$ Blood urea nitrogen (minimum) & 0.15 & -2.23 \\
$\quad$ Urine output & & 5.22 \\
Imbalanced & 0.09 & 0.02 \\
$\quad$ Blood urea nitrogen (minimum) & -3.59 \\
$\quad$ Urine output & & -102 \\
\hline
\end{tabular}

Similar to the MIMIC-III results, the IRT analyses of the eICU both MIMIC-III and eICU were not discriminatory (slopes of showed that BUN was a highly discriminating feature whereas urine output was not (Table 5). In fact, many of the features for $<0.35[52])$.

Table 5. Electronic intensive care unit feature parameters.

\begin{tabular}{lcc}
\hline Feature parameter & Slope & Location \\
\hline Balanced & 1.55 & -0.33 \\
$\quad$ Blood urea nitrogen (minimum) & 0.04 & -1.19 \\
$\quad$ Urine output & & -0.1 \\
Imbalanced & 1.49 & -1.39 \\
$\quad$ Blood urea nitrogen (minimum) & 0.03 & - \\
$\quad$ Urine output & & \\
\hline
\end{tabular}

\section{Classification Results}

Checking the K-nearest neighbors grid warranted using Manhattan distancing and 27 nearest neighbors for MIMIC-III and Manhattan distancing with 19 neighbors for eICU. The neural network grid search results returned an optimum learning rate of 0.001 , activation function softmax, and a number of hidden nodes, 15 for MIMIC-III and 17 for eICU.

Traditional metrics of accuracy, precision, recall, F1, and AUC are presented for MIMIC-III in Table 6. 
Table 6. Medical Information Mart for Intensive Care III classification performance in traditional metrics.

\begin{tabular}{|c|c|c|c|c|c|c|}
\hline Metric & $\mathrm{LR}^{\mathrm{a}}(\%)$ & $\operatorname{LDA}^{\mathrm{b}}(\%)$ & $\mathrm{KNN}^{\mathrm{c}}(\%)$ & $\mathrm{DT}^{\mathrm{d}}(\%)$ & $\mathrm{NB}^{\mathrm{e}}(\%)$ & $\mathrm{NN}^{\mathrm{f}}(\%)$ \\
\hline \multicolumn{7}{|l|}{ Balanced } \\
\hline Accuracy & 75.3 & 75.0 & 67.2 & 70.9 & 70.4 & 76.1 \\
\hline Precision & 75.8 & 75.6 & 69.3 & 71.1 & 79.5 & 75.6 \\
\hline Recall & 74.3 & 73.8 & 61.8 & 70.6 & 54.9 & 77.2 \\
\hline $\mathrm{F} 1$ & 75.0 & 74.7 & 65.3 & 70.8 & 64.9 & 76.4 \\
\hline $\mathrm{AUC}^{\mathrm{g}}$ & 75.3 & 75.0 & 67.2 & 70.9 & 70.4 & 76.5 \\
\hline \multicolumn{7}{|l|}{ Imbalanced } \\
\hline Accuracy & 78.3 & 77.9 & 72.8 & 73.7 & 75.3 & 80.5 \\
\hline Precision & 73.3 & 73.8 & 63.1 & 60.6 & 67.7 & 72.7 \\
\hline Recall & 54.8 & 52.1 & 44.4 & 60.6 & 49.6 & 66.6 \\
\hline $\mathrm{F} 1$ & 62.7 & 61.1 & 52.2 & 60.6 & 57.3 & 69.5 \\
\hline AUC & 72.4 & 71.4 & 65.7 & 70.9 & 68.9 & 76.9 \\
\hline
\end{tabular}

${ }^{\mathrm{a}} \mathrm{LR}$ : logistic regression.

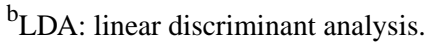

${ }^{\mathrm{c}} \mathrm{KNN}$ : K-nearest neighbor.

${ }^{\mathrm{d}} \mathrm{DT}$ : decision tree.

e NB: naive Bayes.

${ }^{\mathrm{f}} \mathrm{NN}$ : neural network.

${ }^{\mathrm{g}} \mathrm{AUC}$ : area under the curve.

In both the balanced and imbalanced MIMIC-III data sets, the neural network outperformed the other classifiers (balanced: accuracy was $76.1 \%$ and imbalanced: accuracy was $80.5 \%$ ) using traditional metrics. It is worth highlighting the role an imbalanced data set has on an increased accuracy and a reduction in precision, recall, and $\mathrm{F} 1$.

Table 7 shows our proposed method of demonstrating accuracy as a function of CDI. The metric used in Table 7 is accuracy as $\mathrm{F} 1$, recall, and precision were undefined in the extreme negative (where features were predominantly 0 ), and no cases of death existed by which to divide. A parabolic relationship existed in the accuracy level and the strata values, where those more distant from the strata bin $=0$ were more likely to be classified correctly. ML researchers should be most interested in the problematic cases CDI bin 0.0 and where we observe that all classifiers

struggle with prediction. These results suggest that even if a classifier outperforms its counterparts as shown in the traditional metrics of Table 6 (eg, neural network), it may be surpassed in the more fine-grained approach shown in Table 7 (eg, naive Bayes algorithm within the +1.5 CDI bin of the balanced data set).

In both the balanced and the imbalanced eICU data sets (Table 8 ), the neural network outperformed the other classifiers using traditional metrics. Similar to the MIMIC-III findings, the imbalanced data set resulted in increased accuracy and decreased precision, recall, and F1.

Table 9 shows our alternative method of demonstrating accuracy as a function of CDI. Cases that were more distant from the strata bin $=0$ were more likely to be classified correctly. 
Table 7. Item response theory-based Medical Information Mart for Intensive Care III mortality prediction accuracy stratified by classification difficulty index.

\begin{tabular}{|c|c|c|c|c|c|c|c|}
\hline Number of cases & $\mathrm{CDI}^{\mathrm{a}}$ & $\mathrm{LR}^{\mathrm{b}}(\%)$ & $\operatorname{LDA}^{\mathrm{c}}(\%)$ & $\mathrm{KNN}^{\mathrm{d}}(\%)$ & $\mathrm{DT}^{\mathrm{e}}(\%)$ & $\mathrm{NB}^{\mathrm{f}}(\%)$ & $\mathrm{NN}^{\mathrm{g}}(\%)$ \\
\hline \multicolumn{8}{|l|}{ Balanced } \\
\hline 1 & 2.5 & 100.0 & 100.0 & 100.0 & 100.0 & 100.0 & 100.0 \\
\hline 13 & 2.0 & 92.3 & 92.3 & 84.6 & 92.3 & 92.3 & 92.3 \\
\hline 316 & 1.5 & 90.2 & 88.2 & 80.4 & 80.4 & 89.2 & 88.3 \\
\hline 1884 & 1.0 & 75.6 & 74.9 & 68.2 & 68.8 & 68.4 & 77.0 \\
\hline 1321 & 0.5 & 70.5 & 70.6 & 63.5 & 65.9 & 65.4 & 71.1 \\
\hline 952 & 0.0 & 72.0 & 72.4 & 62.8 & 68.8 & 66.2 & 73.9 \\
\hline 1346 & -0.5 & 70.9 & 70.6 & 60.4 & 67.1 & 63.7 & 72.1 \\
\hline 1955 & -1.0 & 77.0 & 77.1 & 70.9 & 75.4 & 75.2 & 78.3 \\
\hline 288 & -1.5 & 94.8 & 94.8 & 83.3 & 91.0 & 95.5 & 94.5 \\
\hline 3 & -2.0 & 100.0 & 100.0 & 100.0 & 100.0 & 100.0 & 100.0 \\
\hline \multicolumn{8}{|l|}{ Imbalanced } \\
\hline 1 & 2.5 & 100.0 & 100.0 & 100.0 & 100.0 & 100.0 & 100.0 \\
\hline 30 & 2.0 & 93.3 & 93.3 & 76.7 & 73.3 & 93.3 & 93.3 \\
\hline 571 & 1.5 & 77.4 & 75.7 & 64.1 & 71.1 & 77.4 & 78.3 \\
\hline 1886 & 1.0 & 70.6 & 70.3 & 63.9 & 65.0 & 64.6 & 73.3 \\
\hline 1537 & 0.5 & 76.3 & 75.5 & 67.3 & 71.2 & 72.7 & 79.7 \\
\hline 1251 & 0.0 & 78.7 & 78.0 & 75.6 & 74.5 & 76.8 & 80.3 \\
\hline 2794 & -0.5 & 75.0 & 74.5 & 71.0 & 72.1 & 72.3 & 78.4 \\
\hline 2722 & -1.0 & 88.3 & 88.3 & 85.0 & 83.3 & 87.1 & 89.1 \\
\hline 325 & -1.5 & 99.1 & 99.1 & 96.6 & 98.2 & 99.1 & 98.8 \\
\hline
\end{tabular}

${ }^{\mathrm{a}} \mathrm{CDI}$ : classification difficulty index.

${ }^{\mathrm{b}} \mathrm{LR}$ : logistic regression.

${ }^{\mathrm{c}}$ LDA: linear discriminant analysis.

${ }^{\mathrm{d}} \mathrm{KNN}$ : K-nearest neighbor.

${ }^{\mathrm{e}} \mathrm{DT}$ : decision tree.

${ }^{\mathrm{f}} \mathrm{NB}$ : naive Bayes.

${ }^{\mathrm{g} N} \mathrm{~N}$ : neural network. 
Table 8. Electronic intensive care unit classification performance in traditional metrics.

\begin{tabular}{|c|c|c|c|c|c|c|}
\hline Metric & $\operatorname{LR}^{\mathrm{a}}(\%)$ & $\operatorname{LDA}^{\mathrm{b}}(\%)$ & $\mathrm{KNN}^{\mathrm{c}}(\%)$ & $\mathrm{DT}^{\mathrm{d}}(\%)$ & $\mathrm{NB}^{\mathrm{e}}(\%)$ & $\mathrm{NN}^{\mathrm{f}}(\%)$ \\
\hline \multicolumn{7}{|l|}{ Balanced } \\
\hline Accuracy & 77.9 & 77.4 & 67.2 & 76.7 & 66.6 & 84.7 \\
\hline Precision & 77.9 & 78.1 & 67.9 & 76.7 & 73.7 & 84.5 \\
\hline Recall & 77.9 & 76.3 & 65.3 & 76.8 & 51.6 & 84.9 \\
\hline $\mathrm{F} 1$ & 77.8 & 77.2 & 66.6 & 76.7 & 60.7 & 84.7 \\
\hline$A U C^{g}$ & 77.9 & 77.4 & 67.2 & 77.1 & 66.6 & 85.9 \\
\hline \multicolumn{7}{|l|}{ Imbalanced } \\
\hline Accuracy & 78.0 & 80.1 & 73.6 & 81.6 & 73.3 & 89.5 \\
\hline Precision & 73.6 & 75.1 & 64.1 & 72.1 & 62.0 & 84.7 \\
\hline Recall & 62.1 & 60.2 & 47.2 & 72.9 & 51.5 & 83.5 \\
\hline $\mathrm{F} 1$ & 67.4 & 66.8 & 54.4 & 72.5 & 56.3 & 84.1 \\
\hline AUC & 75.5 & 75.1 & 67.0 & 79.3 & 67.9 & 87.8 \\
\hline
\end{tabular}

${ }^{\mathrm{a}} \mathrm{LR}$ : logistic regression.

${ }^{b}$ LDA: linear discriminant analysis.

${ }^{\mathrm{c}} \mathrm{KNN}$ : K-nearest neighbor.

${ }^{\mathrm{d} D T}$ : decision tree.

${ }^{\mathrm{e}} \mathrm{NB}$ : naive Bayes.

${ }^{\mathrm{f}} \mathrm{NN}$ : neural network.

${ }^{\mathrm{g}}$ AUC: area under the curve. 
Table 9. Item response theory-based electronic intensive care unit mortality prediction accuracy stratified by classification difficulty index.

\begin{tabular}{|c|c|c|c|c|c|c|c|}
\hline Number of cases & $\mathrm{CDI}^{\mathrm{a}}$ & $\mathrm{LR}^{\mathrm{b}}(\%)$ & $\operatorname{LDA}^{\mathrm{c}}(\%)$ & $\mathrm{KNN}^{\mathrm{d}}(\%)$ & $\mathrm{DT}^{\mathrm{e}}(\%)$ & $\mathrm{NB}^{\mathrm{f}}(\%)$ & $\mathrm{NN}^{\mathrm{g}}(\%)$ \\
\hline \multicolumn{8}{|l|}{ Balanced } \\
\hline 2 & 3.0 & 100.0 & 100.0 & 100.0 & 50.0 & 100.0 & 100.0 \\
\hline 61 & 2.5 & 82.0 & 82.0 & 75.4 & 78.7 & 86.9 & 85.2 \\
\hline 160 & 2.0 & 81.3 & 82.5 & 75.0 & 76.3 & 81.9 & 83.4 \\
\hline 621 & 1.5 & 86.2 & 86.8 & 74.5 & 79.2 & 83.7 & 87.9 \\
\hline 3167 & 1.0 & 83.7 & 82.9 & 72.1 & 78.3 & 66.3 & 85.4 \\
\hline 4998 & 0.5 & 74.0 & 72.7 & 64.7 & 73.1 & 55.2 & 80.9 \\
\hline 4776 & 0.0 & 70.9 & 70.1 & 58.5 & 71.5 & 57.3 & 80.0 \\
\hline 3864 & -0.5 & 73.8 & 74.5 & 63.3 & 74.4 & 67.4 & 84.3 \\
\hline 2858 & -1.0 & 85.4 & 85.5 & 74.4 & 84.8 & 83.1 & 91.8 \\
\hline 1183 & -1.5 & 92.5 & 92.6 & 84.3 & 91.7 & 91.9 & 96.4 \\
\hline 240 & -2.0 & 97.1 & 97.1 & 91.7 & 95.8 & 96.3 & 97.9 \\
\hline 10 & -2.5 & 100.0 & 100.0 & 100.0 & 100.0 & 100.0 & 100.0 \\
\hline \multicolumn{8}{|l|}{ Imbalanced } \\
\hline 6 & 3.0 & 66.7 & 83.3 & 83.3 & 66.6 & 66.6 & 83.3 \\
\hline 58 & 2.5 & 82.8 & 81.0 & 69.0 & 75.9 & 87.9 & 84.5 \\
\hline 215 & 2.0 & 79.1 & 78.6 & 67.0 & 72.6 & 76.3 & 82.3 \\
\hline 1369 & 1.5 & 79.8 & 79.0 & 65.4 & 75.2 & 72.8 & 85.7 \\
\hline 4776 & 1.0 & 72.2 & 72.4 & 61.6 & 74.8 & 58.4 & 83.9 \\
\hline 6657 & 0.5 & 67.3 & 67.0 & 72.1 & 57.3 & 57.3 & 83.1 \\
\hline 7068 & 0.0 & 76.4 & 76.9 & 70.0 & 78.8 & 70.3 & 88.5 \\
\hline 6396 & -0.5 & 87.1 & 87.3 & 83.2 & 87.3 & 83.4 & 93.7 \\
\hline 4265 & -1.0 & 94.8 & 95.0 & 92.0 & 94.3 & 92.7 & 97.7 \\
\hline 1763 & -1.5 & 98.0 & 98.0 & 97.1 & 97.9 & 97.3 & 99.4 \\
\hline 317 & -2.0 & 99.1 & 99.1 & 98.4 & 98.4 & 98.4 & 99.1 \\
\hline 20 & -2.5 & 100.0 & 100.0 & 100.0 & 100.0 & 100.0 & 100.0 \\
\hline
\end{tabular}

${ }^{\mathrm{a}} \mathrm{CDI}$ : classification difficulty index.

${ }^{\mathrm{b}} \mathrm{LR}$ : logistic regression.

${ }^{\mathrm{C}}$ LDA: linear discriminant analysis.

${ }^{\mathrm{d}} \mathrm{KNN}$ : K-nearest neighbor.

${ }^{\mathrm{e}} \mathrm{DT}$ : decision tree.

${ }^{\mathrm{f}} \mathrm{NB}$ : naive Bayes.

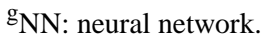

\section{Effect Testing}

The CDI group sizes at the extreme ends were too small and were collapsed into the next level down for each data set. Tests of the effects of MIMIC-III are reported in Table 10 and Figure

The MIMIC-III balanced data showed significantly better accuracies for the more peripheral than central CDI bins. K-nearest neighbors and decision tree were the poorest classifiers. Although there was a small significant interaction effect, by and large, the main effects were borne out. 
Table 10. Tests of the effects of classification difficulty index, classifier, and their interaction for the Medical Information Mart for Intensive Care III data set.

\begin{tabular}{|c|c|c|c|}
\hline \multirow[t]{2}{*}{ Effect } & \multicolumn{2}{|l|}{ Significance } & \multirow[t]{2}{*}{$\begin{array}{l}\text { Significant paired comparisons }(P<.001 \text {; higher accuracies } \\
\text { listed first })\end{array}$} \\
\hline & $F$ test (df) & $P$ value & \\
\hline \multicolumn{4}{|l|}{ Balanced } \\
\hline $\mathrm{CDI}^{\mathrm{a}}$ & $123(6,48456)$ & $<.001$ & $\begin{array}{ll}- & -1.5 \mathrm{vs}-1.0,-0.5,0.0 \\
- & -1.0 \mathrm{vs}-.05,0.0 \\
\text { - } & +1.0 \mathrm{vs}+0.5,0.0 \\
& +1.5 \mathrm{vs}+1.0,+0.5,0.0\end{array}$ \\
\hline $\mathrm{ML}^{\mathrm{b}}$ classifier & $52(5,48456)$ & $<.001$ & $\begin{array}{l}\text { - } \mathrm{LR}^{\mathrm{c}}, \mathrm{LDA}^{\mathrm{d}}, \mathrm{NB}^{\mathrm{e}}, \mathrm{NN}^{\mathrm{f}} \text { vs KNN }{ }^{\mathrm{g}}, \mathrm{DT}^{\mathrm{h}} \\
\text { - } \\
\mathrm{DT} \text { vs KNN }\end{array}$ \\
\hline CDI $\times$ ML classifier & $2(30,48456)$ & $<.001$ & $\begin{array}{ll}- & -1.5: \text { LR, LDA, NB, NN, DT vs KNN } \\
- & -1.0: \text { LR, LDA, NB, NN, DT vs KNN } \\
- & -0.5: \text { LR, LDA, DT, NN vs NB, KNN } \\
- & 0.0: \text { LR, LDA, DT, NN vs NB, KNN } \\
- & +0.5: \text { LR, LDA, NN vs NB, KNN, DT } \\
- & +1.0: \text { LR, LDA, NN vs NB, KNN, DT } \\
- & +1.5: \text { LR, LDA, NB, NN vs KNN DT }\end{array}$ \\
\hline \multicolumn{4}{|l|}{ Imbalanced } \\
\hline CDI & $314(6,72660)$ & $<.001$ & $\begin{array}{ll}- & -1.5 \mathrm{vs}-1.0,-0.5,0.0 \\
\text { - } & -1.0 \mathrm{vs}-.05,0.0 \\
\text { - } & 0.0 \mathrm{vs}-0.5,+0.5,+1.0 \\
\text { - } & +0.5 \mathrm{vs}+1.0 \\
\text { - } & +1.5 \mathrm{vs}+1.0\end{array}$ \\
\hline ML classifier & $12(5,72660)$ & $<.001$ & - $\quad$ LR, LDA, NB, NN vs KNN, DT \\
\hline $\mathrm{CDI} \times \mathrm{ML}$ classifier & $2(30,72660)$ & .004 & $\begin{array}{ll}- & -1.5: \text { no differences } \\
- & -1.0: \text { LR, LDA, NB, NN vs KNN, DT } \\
\text { - } & -0.5: \text { LR, LDA, NN vs NB, KNN, DT } \\
\text { - } & 0.0: \text { NN vs DT }\end{array}$ \\
\hline
\end{tabular}

${ }^{\mathrm{a}} \mathrm{CDI}$ : classification difficulty index.

${ }^{\mathrm{b}} \mathrm{ML}$ : machine learning.

${ }^{\mathrm{c}} \mathrm{LR}$ : logistic regression.

${ }^{\mathrm{d}}$ LDA: linear discriminant analysis.

${ }^{\mathrm{e}} \mathrm{NB}$ : naive Bayes.

${ }^{\mathrm{f}} \mathrm{NN}$ : neural network.

${ }^{\mathrm{K} N N} \mathrm{~K}$ : K-nearest neighbor.

${ }^{\mathrm{h}} \mathrm{DT}$ : decision tree. 
Figure 4. Medical Information Mart for Intensive Care (MIMIC) III generalized linear mixed model (GLMM) accuracy results; machine learning classifier against CDI for (A) balanced and (B) imbalanced data. DT: decision tree; KNN: K-nearest neighbors; LDA: linear discriminant analysis; LR: logistic regression; NB: naive Bayes; NN: neural network.

A

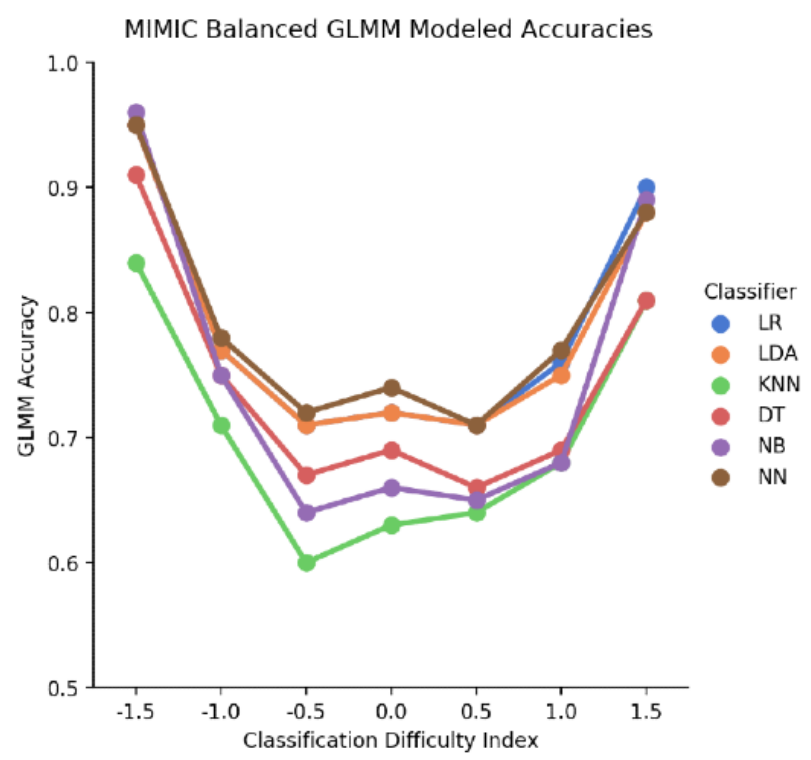

The MIMIC-III imbalanced data set showed that at the healthier end of the CDI continuum, more peripheral cases were accurately classified. This was not the case at the central and unhealthier end of the continuum. Like the balanced data set, K-nearest neighbors and decision tree were the poorest
B

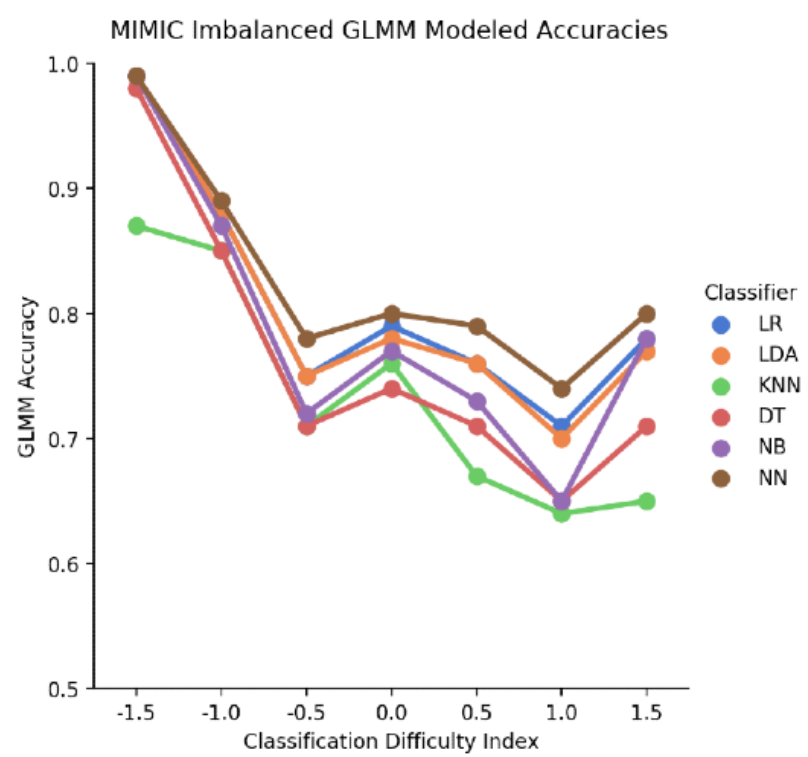

classifiers. Although the interaction was significant, most of the paired comparisons supported the main effect findings.

Tests of the effects from eICU are reported in Table 11 and Figure 5. 
Table 11. Tests of the effects of classification, classifier, and their interaction for the electronic intensive care unit data set.

\begin{tabular}{|c|c|c|c|}
\hline \multirow[t]{2}{*}{ Effect } & \multicolumn{2}{|l|}{ Significance } & \multirow[t]{2}{*}{$\begin{array}{l}\text { Significant paired comparisons }(P<.001 \text {; higher accuracies } \\
\text { listed first })\end{array}$} \\
\hline & $F$ test $(\mathrm{df})$ & $P$ value & \\
\hline \multicolumn{4}{|l|}{ Balanced } \\
\hline $\mathrm{CDI}^{\mathrm{a}}$ & $382(8,131586)$ & $<.001$ & $\begin{array}{ll}\text { - } & -2.0 \mathrm{vs}-1.5,-1.0,-0.5,0.0 \\
\text { - } & -1.5 \mathrm{vs}-1.0,-0.5,0.0 \\
\text { - } & +1.0 \mathrm{vs}-.05,0.0 \\
\text { - } & +1.5 \mathrm{vs}+1.0,0.0 \\
\mathbf{} & +2.0 \mathrm{vs}+0.5,0.0\end{array}$ \\
\hline $\mathrm{ML}^{\mathrm{b}}$ classifier & $58(5,131586)$ & $<.001$ & - $\quad \mathrm{NN}^{\mathrm{c}}$ vs LR ${ }^{\mathrm{d}}, \mathrm{LDA}^{\mathrm{e}}, \mathrm{DT}^{\mathrm{f}} \mathrm{vs} \mathrm{NB}^{\mathrm{g}}$ vs KNN \\
\hline $\mathrm{CDI} \times \mathrm{ML}$ classifier & $9(40,131586)$ & $<.001$ & 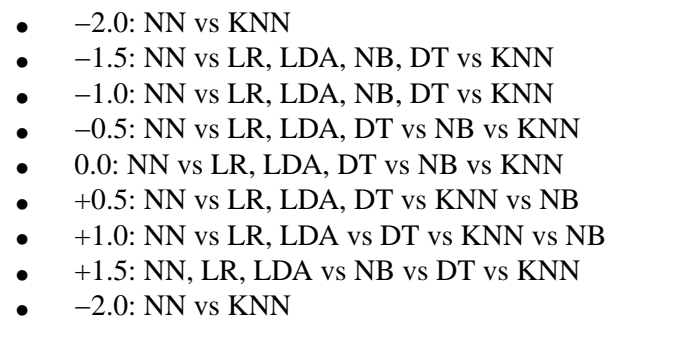 \\
\hline \multicolumn{4}{|l|}{ Imbalanced } \\
\hline Difficulty CDI & $1138(8,197406)$ & $<.001$ & $\begin{array}{ll}\text { - } & -2.0 \mathrm{vs}-1.0,-0.5,0.0 \\
\text { - } & -1.5 \mathrm{vs}-1.0,-0.5,0.0 \\
\text { - } & -1.0 \mathrm{vs}-.05,0.0 \\
\text { - } & -0.5 \mathrm{vs} 0.0 \\
\text { - } & 0.0 \mathrm{vs}+0.5,+1.0 \\
\text { - } & +1.0 \mathrm{vs}+0.5 \\
\text { - } & +1.5 \mathrm{vs}+0.5,+1.0 \\
\text { - } & +2.0 \mathrm{vs}+1.0,+0.5\end{array}$ \\
\hline ML classifier & $28(5,197406)$ & $<.001$ & - $\quad \mathrm{NN}$ vs LR, LDA vs DT vs NB, KNN \\
\hline $\mathrm{CDI} \times \mathrm{ML}$ classifier & $4(40,197406)$ & $<.001$ & $\begin{array}{ll}- & -2.0: \text { no differences } \\
\text { - } & -1.5: \text { NN vs LR, LDA, NB, KNN, DT } \\
\text { - } & -0.5: \text { NN vs LR, LDA, DT vs KNN, NB } \\
\text { - } & \text { 0.0: NN vs LR, LDA, DT vs KNN, NB } \\
\text { - } & \text { +0.5: NN vs LR, LDA vs DT vs KN, NB } \\
\text { - } & \text { 1.0: NN vs LR, LDA, DT vs KNN, NB } \\
\text { - } & \text { +2.0: NN, LR vs LDA vs DT, NB vs KNN } \\
\text { 2. KNN }\end{array}$ \\
\hline
\end{tabular}

${ }^{\mathrm{a}} \mathrm{CDI}$ : classification difficulty index.

${ }^{\mathrm{b}} \mathrm{ML}$ : machine learning.

${ }^{\mathrm{c}} \mathrm{NN}$ : neural network.

${ }^{\mathrm{d}} \mathrm{LR}$ : logistic regression.

${ }^{\mathrm{e}}$ LDA: linear discriminant analysis.

${ }^{\mathrm{f}} \mathrm{DT}$ : decision tree.

$\mathrm{g}_{\mathrm{NB}}$ : naive Bayes.

${ }^{\mathrm{h}} \mathrm{KNN}$ : K-nearest neighbor. 
Figure 5. Electronic intensive care unit (eICU) generalized linear mixed model (GLMM) accuracy results; machine learning classifier against CDI for (A) balanced and (B) imbalanced data. DT: decision tree; KNN: K-nearest neighbors; LDA: linear discriminant analysis; LR: logistic regression; NB: naive Bayes; NN: neural network.

A

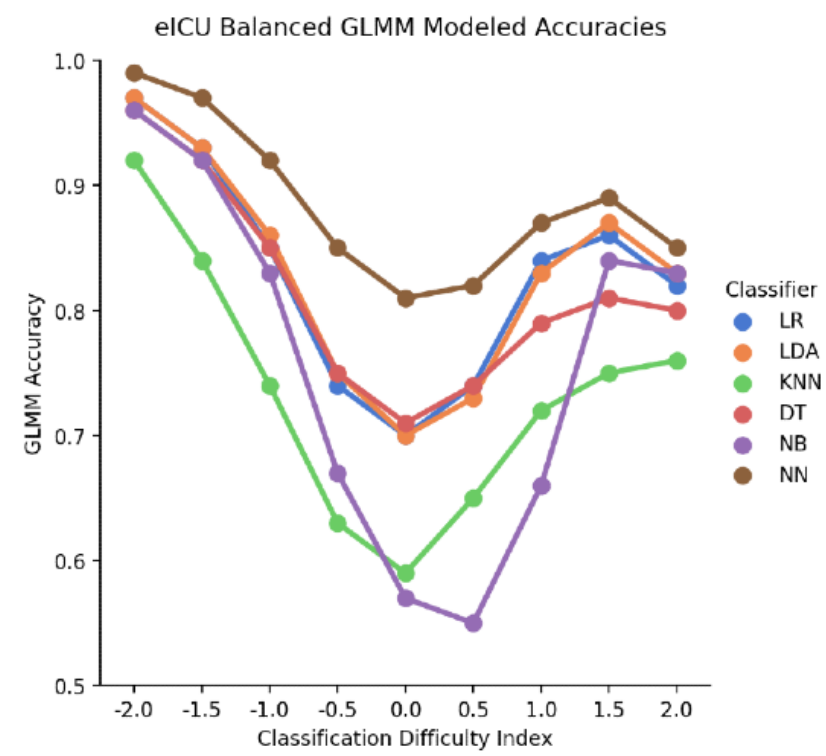

For the eICU balanced data set, moving away from the central bin showed significantly better accuracy, except at the +2.0 level, which was similar to the $+1.5 \mathrm{ML}$ classifier estimated means showed that the neural network had significantly better accuracy than all other classifiers. The overall interaction effect was significant, but the paired comparisons were similar to the main effects.

For the eICU imbalanced data set, more peripheral cases were accurately classified at the healthier end of the distribution, whereas there was only a slight improvement at the unhealthier end. Similar to the other analyses, the neural network showed the best classification accuracy. Although the overall interaction was significant, the neural network continued to be the best classifier.

\section{Discussion}

\section{Principal Findings}

The results generally supported the hypothesis that cases with more extreme IRT-based CDI values are more likely to be correctly classified than cases with more central CDI values. This provides a unique manner to evaluate the utility of ML classifiers in a health context. We were able to demonstrate that ML classifiers performed similarly for the extreme cases, whereas for the centrally located cases, there were more differences between classifiers. Thus, ML classifiers can be evaluated based on their relative performance with cases of varying difficulty.

Although these were the general results, there were several specific findings that are worth noting. First, the neural network classifier was the best across all situations. The logistic regression and linear discriminant analysis classifiers were close to the second-best classifiers, whereas K-nearest neighbors almost always performed the worst. It is possible, as found in

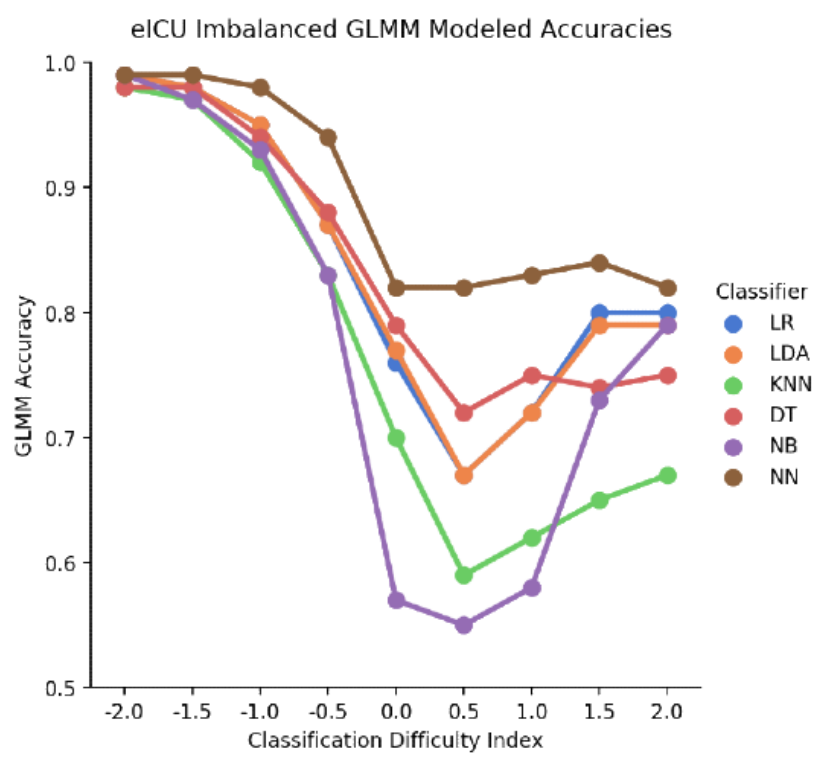

this study, that classifiers may turn out to be consistent over all levels of difficulty. However, owing to the unique characteristics of both data sets and classifiers selected, some algorithms may yield better results at various levels of case difficulty in other samples.

It was also clear that the peripheral-central trend of correct classification was most closely adhered to for cases with negative CDI values (ie, at the healthier end of the CDI distribution), and this trend was particularly pronounced with the imbalanced ( $2 / 3$ nondeath) data sets. We adopted this modest imbalance in this research to detect trends such as these. This finding is pertinent to ML training protocols in that it is best to train them on balanced data sets before running them on imbalanced ones. There is a clear training effect toward negative CDI or the majority class in our case.

On the basis of the IRT analysis results, easier- and harder-to-classify cases were identified. This has implications for research and clinical practice. Once the cases have been identified, other information gathered from their patient-specific data may provide clues about why they are easier or harder to classify, diagnose, or treat. The features themselves that have varying weighted importance in the indexing process can be examined to assess for any differences in a patient's CDI, that is, not just how many they got wrong but which they got wrong or correct to justify their position in eluding an ML classifier.

As an example of how one could examine more closely the problematic patients, we selected the neural network accuracies for each case in the 0 CDI bin in the MIMIC-III balanced data set. This provided 952 cases, 704 (73.9\%) were correctly classified and 248 (26.1\%) were not. A series of chi-square analyses were conducted using the in and out-of-range coding for each of the features crossed with accuracy. Not surprisingly, these cases did not differ on most of the features; the only ones with differences were $\mathrm{WBC} \max \left(\chi_{1}^{2}=5.6 ; P=.02\right)$, where those 
who were more accurately classified had out-of-range scores; bilirubin $\max \left(\chi_{1}^{2}=4.2 ; P=.04\right)$, where those who were more accurately classified had normal scores; and mean heart rate $\left(\chi_{1}^{2}=7.1 ; P=.008\right.$, where those who were more accurately classified had normal scores. Using an approach like this can assist in determining which features in more problematic cases may be differentiated.

\section{Relationship With Previous Work}

An IRT analysis can assist in providing a better understanding of why the classification process works well or falls short on the set of features and cases under investigation. This moves the field closer to having interpretable and explainable results $[53,54]$. Recent research with another ICU data set also argues about the importance of explainable processes as well as results [55]. Early research into ML focused on knowledge as an outcome and adopted an informal approach to evaluation. As the field has progressed, the focus shifted to large data sets, mathematical formulae, single evaluation metrics, and statistics, which has impoverished the discipline [22]. "Choosing performance metrics and confidence estimation methods blindly and applying them without any regard for their meaning and the conditions governing them, is not a particularly interesting endeavor and can result in dangerously misleading conclusions" [9].

\section{Limitations and Future Research}

Limitations of this research include the fact that classifiers showcased here were not exhaustive, only ICU data sets were used, and converting an out-of-range laboratory value as either in range $=0$ or out of range $=1$ is reductive. Although this is true, the purpose of this study is to demonstrate a new evaluation metric using a basic 2PL model with binary data.

There are several ways to extend this work. Future research calls for (1) applying this method to other data sets to generalize its use, (2) using polytomous IRT models (eg, $0=$ in range, $1=$ somewhat out of range, and $2=$ very out of range) for more fine-grained case CDI scoring, (3) using multidimensional IRT models to obtain CDIs on $>1$ underlying dimension, and (4) using this approach to compare human versus machine classification accuracy across case difficulty. We can extend the intersection of ML with clinical medicine if we liken a physician to an ML classifier using feature data. It would be particularly interesting to compare case accuracies based on traditional ML versus clinical classifiers for cases of varying difficulty using an approach similar to that demonstrated in this study. Identifying which cases clinical classifiers are better suited to address, and which cases should be offloaded to an automated system allows for the optimal use of scarce resources. As clinical expertise is developed over time, the use of ML algorithms to assist any single individual would be a moving target and would also serve as a source of future research.

Another way to improve the veracity of the findings would be to address the issue of extraneous features. Several of the features in MIMIC-III and eICU had very low $(<0.35)$ discrimination (slope) parameters, suggesting that there was a lot of noise in the cases' CDIs as well as in the ML classifications. It would be a useful exercise to a priori determine the most useful features [5] and then run the analyses outlined in this study using a more refined feature set.

\section{Conclusions}

As more ML methods are investigated in the health care sphere, concerns have risen because of a lack of understanding regarding why they are successful, especially when compared with physician counterparts. This study has suggested an IRT-based methodology as one way to address this issue by examining the case difficulty in a data set that allows for follow-up into possible reasons why cases are or are not classified correctly.

Using the methods described in this study would signal a change in the way we evaluate supervised ML. Adopting them would move the field toward more of an evaluation system that characterizes the entire data set on which the classifiers are being trained and tested. Doing so circumvents the pitfalls associated with 1 classifier being cited as more accurate or more precise and generates a more tailored approach to ML classifier comparisons. In addition, this methodology lends itself well to post hoc inspections of the data as to what makes difficult cases challenging.

The method here presents an intersection of personalized medicine and ML that maintains its explainability and transparency in both feature selection and modeled accuracy, both of which are pivotal to their uptake in the health sphere.

\section{Authors' Contributions}

AK contributed to idea generation, study and method design, literature search, data acquisition (MIMIC-III data set), figures, tables, data analysis, and writing. TK contributed to data analysis, writing, and proofing the manuscript. ZA contributed to data acquisition of eICU data set. JL contributed to proofing and journal selection.

\section{Conflicts of Interest}

None declared.

\section{References}

1. Bergner Y, Dröschler S, Kortemeyer G, Rayyan S, Seaton D, Pritchard D. Model-based Collaborative Filtering Analysis of Student Response Data: Machine-Learning Item Response Theory. In: 5th International Conference on Educational Data Mining. 2012 Presented at: EDM'12; June 19-21, 2012; Chania, Greece. [doi: 10.1007/978-1-4899-7687-1 945] 
2. Lalor J, Wu H, Yu H. Building an Evaluation Scale Using Item Response Theory. In: Proceedings of the 2016 Conference on Empirical Methods in Natural Language Processing. 2016 Presented at: EMNLP'16; November 1-5, 2016; Austin, Texas, USA. [doi: 10.18653/v1/d16-1062]

3. Pliakos K, Joo S, Park J, Cornillie F, Vens C, Van den Noortgate W. Integrating machine learning into item response theory for addressing the cold start problem in adaptive learning systems. Comput Educ 2019 Aug;137:91-103 [FREE Full text] [doi: 10.1016/j.compedu.2019.04.009]

4. Martínez-Plumed F, Prudêncio R, Martínez-Usó A, Hernández-Orallo J. Item response theory in AI: analysing machine learning classifiers at the instance level. Artif Intell 2019 Jun;271:18-42 [FREE Full text] [doi: 10.1016/j.artint.2018.09.004]

5. Kline A, Kline T, Hossein Abad ZS, Lee J. Novel Feature Selection for Artificial Intelligence Using Item Response Theory for Mortality Prediction. In: Proceedings of the 42nd Annual International Conference of the IEEE Engineering in Medicine \& Biology Society. 2020 Presented at: EMBC'20; July 20-24, 2020; Montreal, QC, Canada p. 5729-5732. [doi:

10.1109/EMBC44109.2020.9175403]

6. Jordan MI, Mitchell TM. Machine learning: trends, perspectives, and prospects. Science 2015 Jul 17;349(6245):255-260. [doi: 10.1126/science.aaa8415] [Medline: 26185243]

7. Greiner R, Silver B, Becker S, Groninger M. A review of machine learning at AAAI-87. Mach Learn 1988 Aug;3(1):79-92. [doi: 10.1007/bf00115011]

8. Hossin M, Sulaiman MN. A review on evaluation metrics for data classification evaluations. Int J Data Min Knowl Manag Process 2015 Mar 31;5(2):1-11. [doi: 10.5121/ijdkp.2015.5201]

9. Japkowicz N. Why Question Machine Learning Evaluation Methods? (an Illustrative Review of the Shortcomings of Current Methods). In: 34th AAAI Conference on Artificial Intelligence. 2006 Presented at: AAAI'06; February 7-12, 2006; New York, USA p. 11.

10. Kotthoff L, Gent I, Miguel I. A Preliminary Evaluation of Machine Learning in Algorithm Selection for Search Problems. In: Proceedings of the Combustion Institute. 2011 Presented at: SoCS'11; March 8-11, 2011; Washington, DC. [doi: 10.3233/aic-2012-0533]

11. Singh K. Performance evaluation of five machine learning algorithms and three feature selection algorithms for IP traffic classification. Int J Comput Appl 2011;1:32 [FRE Full text]

12. Srivastava T. 11 Important Model Evaluation Metrics for Machine Learning Everyone Should Know. Commonly Used Machine Learning Algorithms: Data Science. 2019. URL: https://www.analyticsvidhya.com/blog/2019/08/

11-important-model-evaluation-error-metrics/ [accessed 2020-08-25]

13. Sokolova M, Japkowicz N, Szpakowicz S. Beyond accuracy, F-Score and ROC: Performance evaluation of five machine learning algorithms and three feature selection algorithms for IP traffic classification. In: Advances in Artificial Intelligence Systems. Berlin, Heidelberg: Springer; 2006.

14. Santafe G, Inza I, Lozano JA. Dealing with the evaluation of supervised classification algorithms. Artif Intell Rev 2015 Jun 30;44(4):467-508. [doi: 10.1007/s10462-015-9433-y]

15. Stapor K. Evaluating and Comparing Classifiers: Review, Some Recommendations and Limitations. In: International Conference on Computer Recognition Systems. 2017 Presented at: CORES'17; May 22-24, 2017; Polanica Zdroj, Poland. [doi: 10.1007/978-3-319-59162-9_2]

16. Sokolova M, Lapalme G. A systematic analysis of performance measures for classification tasks. Inf Process Manag 2009 Jul;45(4):427-437. [doi: 10.1016/j.ipm.2009.03.002]

17. Drummond C, Japkowicz N. Warning: statistical benchmarking is addictive. Kicking the habit in machine learning. J Exp Theor Artif In 2010 Mar;22(1):67-80. [doi: 10.1080/09528130903010295]

18. Fatima M, Pasha M. Survey of machine learning algorithms for disease diagnostic. J Intell Learn Syst Appl 2017;09(01):1-16. [doi: 10.4236/jilsa.2017.91001]

19. Senders J, Staples P, Karhade AV, Zaki M, Gormley W, Broekman M, et al. Machine learning and neurosurgical outcome prediction: a systematic review. World Neurosurg 2018 Jan;109:476-86.e1. [doi: 10.1016/j.wneu.2017.09.149] [Medline: 28986230]

20. Christodoulou E, Ma J, Collins GS, Steyerberg EW, Verbakel JY, van Calster B. A systematic review shows no performance benefit of machine learning over logistic regression for clinical prediction models. J Clin Epidemiol 2019 Jun;110:12-22. [doi: 10.1016/j.jclinepi.2019.02.004] [Medline: 30763612]

21. Drummond C. Machine Learning as an Experimental Science. AAAI. 2006. URL: https://www.aaai.org/Papers/Workshops/ 2006/WS-06-06/WS06-06-002.pdf [accessed 2020-09-08]

22. Langley P. The changing science of machine learning. Mach Learn 2011 Feb 18;82(3):275-279. [doi: 10.1007/s10994-011-5242-y]

23. Rudin C. Stop explaining black box machine learning models for high stakes decisions and use interpretable models instead. Nat Mach Intell 2019 May 13;1(5):206-215. [doi: 10.1038/s42256-019-0048-x]

24. Cabitza F, Rasoini R, Gensini GF. Unintended consequences of machine learning in medicine. J Am Med Assoc 2017 Aug 8;318(6):517-518. [doi: 10.1001/jama.2017.7797] [Medline: 28727867]

25. Chen JH, Asch SM. Machine learning and prediction in medicine - beyond the peak of inflated expectations. N Engl J Med 2017 Jun 29;376(26):2507-2509 [FREE Full text] [doi: 10.1056/NEJMp1702071] [Medline: 28657867] 
26. Handelman GS, Kok HK, Chandra RV, Razavi AH, Huang S, Brooks M, et al. Peering into the black box of artificial intelligence: evaluation metrics of machine learning methods. AJR Am J Roentgenol 2019 Jan;212(1):38-43. [doi: 10.2214/AJR.18.20224] [Medline: 30332290]

27. Stefanowski J. Dealing with data difficulty factors while learning form imbalanced data. In: Matwin S, Mielniczuk J, editors. Challenges in Computational Statistics and Data Mining. Switzerland: Springer; 2016:333-363.

28. Comfort S, Perera S, Hudson Z, Dorrell D, Meireis S, Nagarajan M, et al. Sorting through the safety data haystack: using machine learning to identify individual case safety reports in social-digital media. Drug Saf 2018 Jun;41(6):579-590 [FREE Full text] [doi: 10.1007/s40264-018-0641-7] [Medline: 29446035]

29. Goetz J, Brenning A, Petschko H, Leopold P. Evaluating machine learning and statistical prediction techniques for landslide susceptibility modeling. Comput Geosci 2015 Aug;81:1-11. [doi: 10.1016/j.cageo.2015.04.007]

30. Olson RS, la Cava W, Orzechowski P, Urbanowicz RJ, Moore JH. PMLB: a large benchmark suite for machine learning evaluation and comparison. BioData Min 2017;10:36 [FREE Full text] [doi: 10.1186/s13040-017-0154-4] [Medline: 29238404]

31. Jeatrakul P, Wong KW, Fung CC. Data cleaning for classification using misclassification analysis. J Adv Comput Intell Intell Inform 2010 Apr 20;14(3):297-302. [doi: 10.20965/jaciii.2010.p0297]

32. Johnson AEW, Pollard TJ, Shen L, Lehman LH, Feng M, Ghassemi M. Data Descriptor: MIMIC-III, a freely accessible critical care database. Sci Data 2016;3:1-9. [doi: 10.1038/sdata.2016.35]

33. Johnson AEW, Mark RG. Real-time mortality prediction in the Intensive Care Unit. 2017 Presented at: AMIA Annu Symp proceedings; 2018; San Francisco p. 994-1003.

34. Pollard TJ, Johnson AE, Raffa JD, Celi LA, Mark RG, Badawi O. The eICU collaborative research database, a freely available multi-center database for critical care research. Sci Data 2018 Sep 11;5:180178 [FREE Full text] [doi: 10.1038/sdata.2018.178] [Medline: 30204154]

35. Clinical Laboratory Tests - Adult Normal Values. Medical Council of Canada. URL: https://mcc.ca/objectives/normal-values/ [accessed 2020-08-25]

36. Fuchs L, Novack V, McLennan S, Celi LA, Baumfeld Y, Park S, et al. Trends in severity of illness on ICU admission and mortality among the elderly. PLoS One 2014;9(4):e93234 [FREE Full text] [doi: 10.1371/journal.pone.0093234] [Medline: 24699251]

37. Lindemark F, Haaland OA, Kvåle R, Flaatten H, Johansson KA. Age, risk, and life expectancy in Norwegian intensive care: a registry-based population modelling study. PLoS One 2015;10(5):e0125907 [FREE Full text] [doi:

10.1371/journal.pone.0125907] [Medline: 26011281]

38. Alam R, Haque M, Haque M. An appraisal of mortality in intensive care unit of a level III military hospital of Bangladesh. Indian J Crit Care Med 2017 Sep;21(9):594-598 [FREE Full text] [doi: 10.4103/ijccm.IJCCM 250 17] [Medline: 28970660]

39. Atramont A, Lindecker-Cournil V, Rudant J, Tajahmady A, Drewniak N, Fouard A, et al. Association of age with short-term and long-term mortality among patients discharged from intensive care units in France. JAMA Netw Open 2019 May 3;2(5):e193215 [FREE Full text] [doi: 10.1001/jamanetworkopen.2019.3215] [Medline: 31074809]

40. Mahmood K, Eldeirawi K, Wahidi MM. Association of gender with outcomes in critically ill patients. Crit Care 2012 May 22;16(3):R92 [FREE Full text] [doi: 10.1186/cc11355] [Medline: 22617003]

41. Teasdale G, Jennett D. Assessment of coma and impaired consciousness. Lancet 1974;304(7872):81-84.

42. Teasdale G, Jennett B. Assessment and prognosis of coma after head injury. Acta Neurochir (Wien) 1976;34(1-4):45-55. [doi: 10.1007/BF01405862] [Medline: 961490]

43. Urine 24-Hour Volume. Penn State Hershey Health Information Library. 2011. URL: http://pennstatehershey.adam.com/ content.aspx?productId=117pid=1gid=003425 [accessed 2020-08-25]

44. Lapum J, Verkuyl M, Garcia W, St-Amant O, Tan A. Vital Sign Measurement Across the Lifespan - 1st Canadian Edition. Ryerson University Pressbooks. 2018. URL: https://pressbooks.library.ryerson.ca/vitalsign/ [accessed 2020-09-08]

45. APACHE II Score. MDCalc. URL: https://www.mdcalc.com/apache-ii-score [accessed 2020-08-25]

46. Understanding Mean Arterial Pressure. Health Line. 2019. URL: https://www.healthline.com/health/mean-arterial-pressure [accessed 2020-08-25]

47. apacheApsVar. eICU Collaborative Research Database. URL: https://eicu-crd.mit.edu/eicutables/apacheapsvar/ [accessed 2020-08-25]

48. Bock R, Aitkin M. EM Solution of the Marginal Likelihood Equations. Psychometrika 1981;46(4):443-459.

49. Scientific Software Inc. 2018. URL: https://ssicentral.com/ [accessed 2020-09-08]

50. West BT, Galecki AT. An overview of current software procedures for fitting linear mixed models. American Statistician 2015;135(2):612-615 [FREE Full text] [doi: 10.1198/tas.2011.11077] [Medline: 23606752]

51. Overview (GENLINMIXED Command). IBM - United States. 2020. URL: https://www.ibm.com/support/knowledgecenter/ SSLVMB_24.0.0/spss/advanced/syn_genlinmixed_overview.html [accessed 2020-02-10]

52. Baker F. Item Response Theory the Basics of Item Response Theory. New York, USA: ERIC Clearinghouse on Assessment and Evaluation; 2001.

53. Bennett C, Miller M. How reliable are the results from functional magnetic resonance imaging? Ann NY Acad Sci 2010 Mar;1191:133-155. [doi: 10.1111/j.1749-6632.2010.05446.x] [Medline: 20392279] 
54. Miller T. Explanation in artificial intelligence: insights from the social sciences. Artif Intell 2019 Feb;267:1-38. [doi: 10.1016/j.artint.2018.07.007]

55. Thorsen-Meyer H, Nielsen AB, Nielsen AP, Kaas-Hansen BS, Toft P, Schierbeck J, et al. Dynamic and explainable machine learning prediction of mortality in patients in the intensive care unit: a retrospective study of high-frequency data in electronic patient records. Lancet Digit Heal 2020 Apr;2(4):e179-e191. [doi: 10.1016/s2589-7500(20)30018-2]

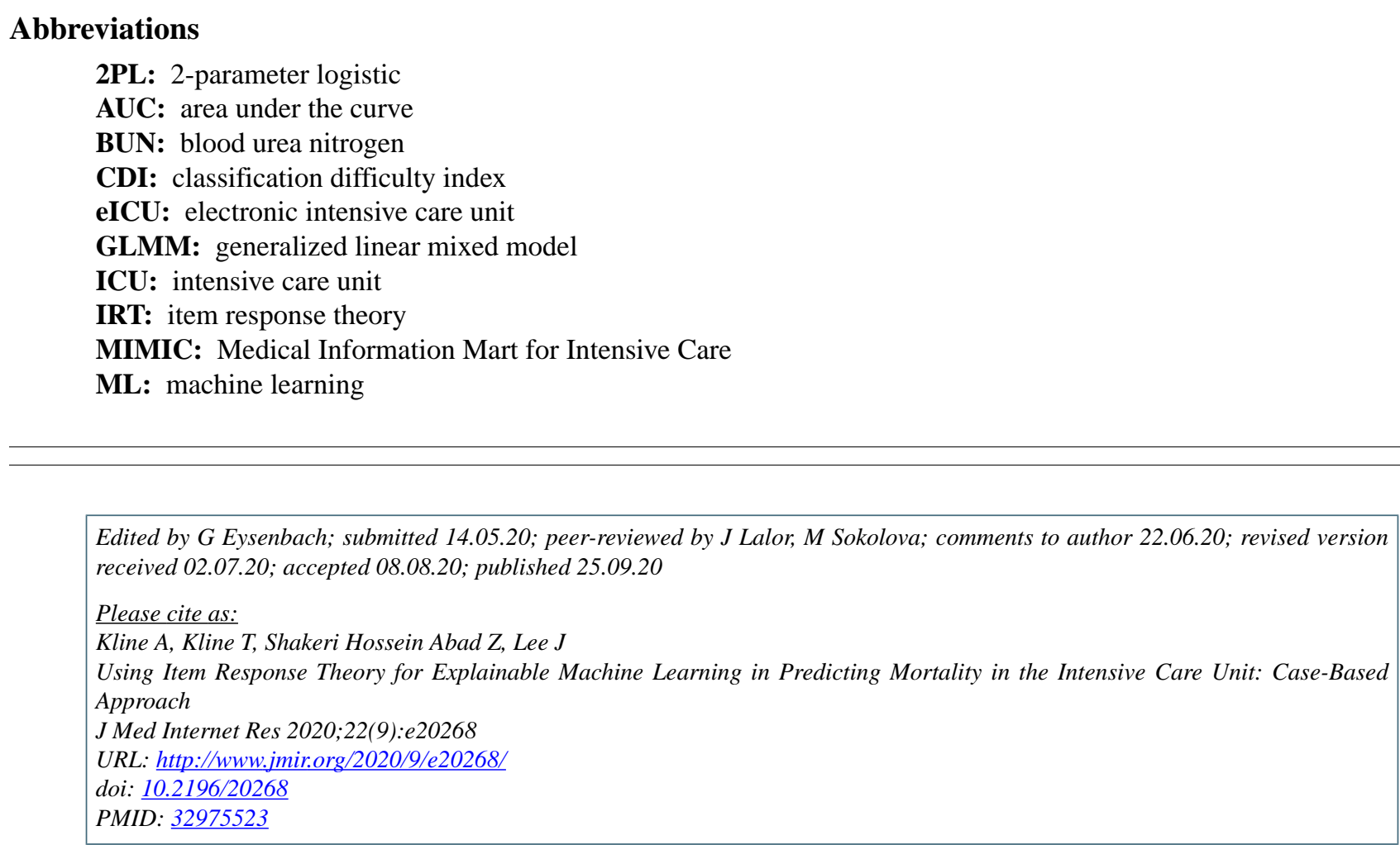

(C)Adrienne Kline, Theresa Kline, Zahra Shakeri Hossein Abad, Joon Lee. Originally published in the Journal of Medical Internet Research (http://www.jmir.org), 25.09.2020. This is an open-access article distributed under the terms of the Creative Commons Attribution License (https://creativecommons.org/licenses/by/4.0/), which permits unrestricted use, distribution, and reproduction in any medium, provided the original work, first published in the Journal of Medical Internet Research, is properly cited. The complete bibliographic information, a link to the original publication on http://www.jmir.org/, as well as this copyright and license information must be included. 